\title{
The effects of mnemonic instruction and list length on serial learning and retention
}

\section{P. S. DELIN, University of Adelaide, Adelaide, South Australia}

Subjects with and without mnemonic instructions learned lists of either 10 or 16 nouns by the anticipation method over four trials. Six we'eks later they relearned the lists. Mnemonic instructions facilitated both learning and relearning, but the relearning effect may have depended on the learning effect. The longer lists were more difficult for both mnemonic and control Ss to learn, but there was no clear difference on relearning. Mnemonic instructions were associated with flattening of the serial-position error curves on both learning and relearning, and with differences in the types of error made by the Ss.

Wallace, Turner, \& Perkins (1957) found that once their Ss had learned a number of lists with the aid of mnemonics they were able to leam lists of widely disparate lengths at very similar rates of presentation, when self-paced. The present writer found, in conversation with a number of professional mnemonists, that they were convinced that length of list made little difference to their level of performance or speed of learning. Since it has long been recognized (Thurstone, 1930) that, for rote learning, item-input-time is related to list length, it seemed worthwhile, as part of a general quest for theoretically suggestive differences between mnemonic and nonmnemonic performance, to compare mnemonic Ss with controls on two different lengths of list.

Earlier experiments by the writer (e.g., Delin, 1969a) had led to the expectation that the use of mnemonic devices would lead to a heightened tendency for errors to take the form of omissions, and would result in flatter serial position error-curves. It was hoped that the present study would confirm these expectations.

Previous research did not justify any clear expectations as to the effects of mnemonic instructions on retention, as compared with their effects on learning. Smith \& Noble (1965) had found much clearer facilitation of retention than of learning, while Olton (1966) had found no facilitation of retention when learning was held constant. In a previous study (Delin, 1969b), the writer found facilitation of retention to increase with completeness of the mnemonic instructions, but it was not possible to tell to what extent this effect depended on facilitation of the original learning. It was hoped that this experiment would clarify the matter.

\section{MATERIALS}

Three lists of concrete nouns were used, a 10-item practise list (List P), a 16-item experimental list (List $L$ ), and a 10-item experimental list (List $\mathrm{S}$ ), which consisted of the first 10 items of List L. All three lists were arranged in such an order as to minimize interitem association, with a view to increasing the numbers of errors made by all Ss, and decreasing the likelihood that control Ss would make use of mnemonic devices. The ordering of the lists was based on interitem association ratings by 20 judges of all possible pairs of the items used.

\section{METHOD}

The Ss, 72 male first-year psychology students aged between 18 and 22 years, were run individually. Each initially learned List $\mathbf{P}$, which was presented by memory drum at a 5 -sec rate for one response-free trial and five anticipation trials, with an intertrial interval of approximately $10 \mathrm{sec}$. On the basis of their error scores in this task, the Ss were assigned to one of six "learning ability" categories. They were then allocated randomly to the four experimental groups, with the restriction that the six "learning ability" categories were to be represented equally in each experimental group.

Having been allocated to one of the four groups, each $S$ learned List $L$ or List $S$, either with mnemonic instructions $(\mathrm{Mn})$ or without them (C). The groups were thus mnemonic-long (Mn-L), mnemonic-short $(\mathrm{Mn} \cdot \mathrm{S})$, control-long (C-L), and control-short (C-S). The lists were presented once at a 6-sec rate, followed by three anticipation trials at the same rate, intertrial interval being 10 sec.

The mnemonic instructions suggested that the Ss should, for each consecutive pair of items in the list, construct an active, vivid image connecting the pair of items, that he should use as many sensory modalities as possible in constructing this image, and that he should make the image extremely bizarre or fantastic. It was further suggested that in the recall phase of the task the $S$ should, when each item appeared in the memory drum window, say to himself, "Now what did I do with that?", and should try to pick the next item out of the image that then sprang to mind. The control Ss were given standard serial anticipation instructions.

After learning the experimental list, each $S$ was cautioned against rehearsal, and given an appointment to return after 6 weeks. Nine Ss failed to return on the appropriate day and the numbers in the relearning groups were thus reduced from 18 to 14 (Mn-L), 16 (C-L), and 15 (C-S), all Mn-S Ss returning.

In the second session, Ss were given four anticipation trials at the same rate as before. Mnemonic Ss were then asked to give examples, where possible, of the images they had used, and whether they had recalled their original images. All Ss were asked whether they had used any learning techniques other than those they had been instructed to use.

\section{RESULTS}

The main results are listed in Table 1 , where short-list error scores have been multiplied by 1.6 to adjust for the difference in opportunity to make errors. The learning error scores do not take into account the first presentation, when Ss were not required to anticipate.

Two-way analyses of variance were carried out separately on the learning and the retention data. For learning, both instructions $(\mathrm{F}=21.4, \mathrm{df}=1 / 68, \mathrm{p}<.01)$ and list length $(\mathrm{F}=15.2, \mathrm{df}=1 / 68, \mathrm{p}<.01)$ showed strong effects. Interaction was insignificant $(\mathrm{F}=1.2)$, a result which does not support the view that list length affects mnemonic performance less than nonmnemonic performance. For relearning, only the instructions had a significant effect $(F=16.7, \mathrm{df}=1 / 59, \mathrm{p}<.01)$, while list length had negligible effect $(F=.8)$. On the other hand, the interaction effect was near significance $(F=3.8$, critical $F$ for $\mathrm{p}<.05=4.0$ )

In an attempt to discover whether the facilitation of retention was dependent on the facilitation of learning, each S's total of correct responses on the learning phase of the task was subtracted from his total of

Table 1

Error Scores on Learning and Relearning: Means and SDs

\begin{tabular}{llccccrr}
\hline & \multicolumn{2}{c}{ Learning } & & \multicolumn{3}{c}{ Relearning } \\
\cline { 2 - 7 } & $\mathrm{n}$ & Mean & SD & n & Mean & SD \\
\hline Mn-L & 18 & 9.8 & 7.28 & 14 & 15.3 & 6.82 \\
Mn-S & 18 & 5.1 & 4.27 & 18 & 14.2 & 12.07 \\
C-L & 18 & 19.3 & 8.04 & 16 & 28.4 & 8.79 \\
C-S & 18 & 11.4 & 6.77 & 15 & 22.7 & 9.88 \\
\hline
\end{tabular}


Table 2

Mean Omission (O) and Commission (C) Error Seores

\begin{tabular}{lrrrrr}
\hline & \multicolumn{2}{c}{ Learning } & & \multicolumn{2}{c}{ Relearning } \\
\cline { 2 - 3 } \cline { 5 - 6 } & \multicolumn{1}{c}{$\mathrm{O}$} & $\mathrm{C}$ & & \multicolumn{1}{c}{$\mathrm{O}$} & \multicolumn{1}{c}{$\mathrm{C}$} \\
\hline $\mathrm{Mn}-\mathrm{L}$ & 6.8 & 3.0 & & 8.8 & 5.7 \\
$\mathrm{Mn}-\mathrm{S}$ & 2.8 & 2.3 & & 8.9 & 5.2 \\
$\mathrm{C}-\mathrm{L}$ & 9.9 & 9.4 & & 12.1 & 16.2 \\
$\mathrm{C}-\mathrm{S}$ & 3.6 & 5.3 & & 10.6 & 12.5 \\
\hline
\end{tabular}

correct responses on the retention phase (short-list scores having been multiplied by 1.6 to make them comparable with long-list scores). The resulting scores should represent performance on relearning with individual differences in learning held constant. The means of the four groups on these derived scores were $10.5(\mathrm{Mn}-\mathrm{L})$, $6.9(\mathrm{Mn}-\mathrm{S}), \quad 6.9(\mathrm{C}-\mathrm{L})$, and $5.0(\mathrm{C}-\mathrm{S})$. Although the differences were in the same direction as those in the learning data, an analysis of variance showed no significant effects (all $F$ ratios being less than 1.0). This analysis then suggested that most of the facilitation of relearning as a result of mnemonic instructions could be attributed to facilitation of the original learning.

Further analyses of variance were carried out separately on the results of the first anticipation trials on both learning and retention, on the grounds that mnemonic learning has been represented as being essentially one-trial (Rock, 1957) and that the first trial of the relearning phase can be seen as representing recall rather than relearning. The results were similar to those obtained from the previous analyses, but the effect of the instructions was rather greater in the first-trial-only analysis of the learning data $(F=24.7, \mathrm{df}=1 / 68, p<.01)$ and rather smaller in the first-trial-only analysis of the relearning data $(F=7.04$, df $=1 / 59$, $p \approx .01)$. These results could be seen as consistent with the suggestions that mnemonic instructions affect the first learning trial more than the later ones, and relearning more than recall.

An analysis of errors in terms of whether they were omission (O) or commission errors (C) was to some degree frustrated by the relative scarcity of errors among the $\mathrm{Mn}-\mathrm{S}$ group, but some suggestive results emerged. For each comparison of a mnemonic group with the equivalent control group, on both learning and relearning, and separately for the first anticipation trial and for later trials, mnemonic Ss consistently made more of their errors in the form of omissions. Table 2 shows the $\mathrm{O}$ and $\mathrm{C}$ means for all groups, on learning and relearning (short-list scores having been multiplied by 1.6 to make them comparable with long-list scores).

In order to evaluate the overall differences in predominant error type between mnemonic and control Ss, the $O$ score of each $S$ was expressed as a percentage of his total error score. Ss who made no errors were excluded from the analysis, and long-list and short-list groups were combined. Mann-Whitney U tests were carried out comparing mnemonic with control Ss. U was tested as a normal deviate, On learning, the difference was significant at the .02 level $(\mathrm{z}=2.49)$, and on relearning, it was significant at the .05 level $(z=1.97)$.

An incidental observation in earlier experiments by the writer had been that mnemonic instructions appeared to flacten the serial position error curve. Figures la and $1 \mathrm{~b}$ show the serial position error curves for the present data, for learning and relearning, respectively. In order to achieve comparability of the curves, the scores were converted using Jensen's (1962) index of relative difficulty. This index involves the correction of the error scores at each position in the list to adjust for between-list differences in total error rate, followed by conversion of the corrected error scores to percentages of total errors. The figures show that for each appropriate pair comparison,

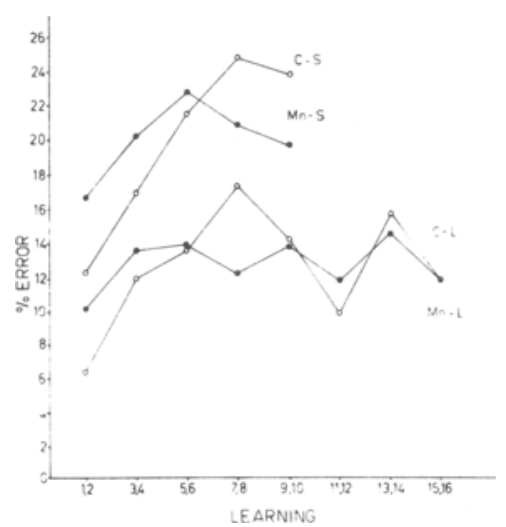

Fig. 1 (a). Jensen Position Error Index.

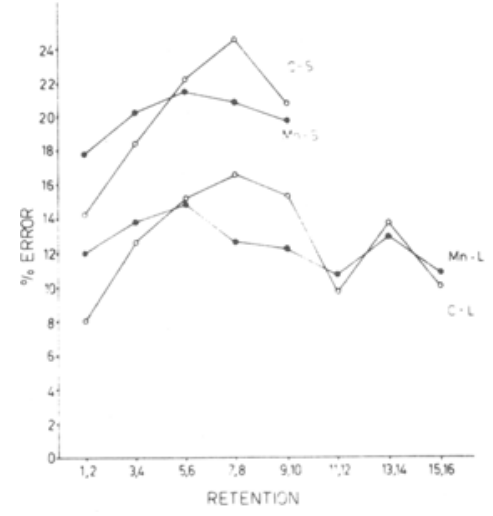

Fig. 1 (b). Jensen Position Error Index. the curve produced by the mnemonic Ss is flatter than that produced by the control Ss.

\section{DISCUSSION}

The absence of an interaction in the learning data between mnemonic instructions and length of list suggests that, under the conditions of this study, list length affects diffculty in the same degree for mnemonic Ss as it does for control Ss. The apparent inconsistency with the results of Wallace et al (1957) might be accounted for by the fact that their Ss, unlike the present ones, were self-paced. It is also worth remarking that the claims of professional mnemonists have generally been made in terms of speed of learning, whereas the present study is concerned with the number of errors made over a fixed number of presentations.

The apparent absence of an effect of list length upon relearning is a little surprising. An examination of the error means on relearning (see Table 1), in the light of the near-significant interaction in the analysis of variance of total errors on relearning, suggests that the absence of a list-length effect is not as clearcut as it might seem. The interaction could be interpreted as suggesting that list length does affect the retention of the control Ss, but not that of the mnemonic Ss.

The analysis of the retention data with the effects of learning held constant had results consistent with those of Olton (1966) in finding that mnemonic instructions had no facilitative effect on retention independent of their effect on learning. One should, however, exercise caution here, as in all cases of failure to reach significance. Furthermore, the results of the analysis of the recall trial alone could be interpreted as evidence that the retention interval was not optimal for the mnemonic Ss. The Ss were asked after the relearning task whether they had recalled their images after they had been presented (during the recall trial) with both the stimulus and the response items. Most said that they had, and that they had used their original images on later relearning trials.

The data were consistent with the accuracy of these reports. It could be that the strength, or integrity, of a mnemonic image is theoretically distinguishable from its availability, and that the mnemonic Ss in this study could not use on the recall trial mnemonic images which were still, so to speak, in store, because the mere presentation of the stimulus item was not sufficient to arouse those images. Given a shorter retention interval, so that the images were at a higher availability level, or a rather longer one, so that all Ss could be expected to make $100 \%$ errors on the recall trial, it might not have appeared that mnemonic 
instructions fail to affect retention independently of their effect upon learning. It should be instructive in this regard to compare mnemonic with control Ss on a recognition test of retention.

The findings on type of error, although only the most general of the comparisons were significant, showed perfect internal consistency in direction. They were also consistent with the subjective reports of professional mnemonists, who report that if they forget their image, or fail to make one, they are often unable to make a response. This result may be relevant to the finding of Montague, Adams, \& Kiess (1966) and Adams \& McIntyre (1967) that Ss who forget their natural language mediators perform worse than Ss who do not report making them.

The observed flattening of the serial position curve is consistent with the implied claims of the mnemonic instructions that the functional stimulus for the mnemonic $S$ is the previous item, and that the difficulty of a given item should depend on its associative relations with the previous item rather than on its position in the list. It would also be possible to see it as consistent with the view that a mnemonic device operates by inducing an artificial meaningfulness in the material, since meaningfulness leads to flattening of the serial position curve (Braun \& Heymann, 1958).

\section{REFERENCFS}

ADAMS, J. A., \& McINTYRE, J. S. Natural language mediation and all-or-none learning. Canadian Journal of Psychology, 1967, 21, 436-449.

BRAUN, H. W., \& HEYMANN, S. P. Meaningfulness of material, distribution of practice, and serial position curves. Journal of Experimental Psychology, 1958, 56, 146-150.

DELIN, P. S. The learning to criterion of a serial list with and without mnemonic instructions. Psychonomic Science, 1969, 16, 4, 169-170.

DELIN. P. S. Learning and retention of English words with successive approximations to a complex mnemonic instruction. Psychonomic Science, $1969,17,2,87-89$.

JENSEN, A. R. Is the serial position curve invariant? British Journal of Psychology, 1962, 53, $159-166$.

MONTAGUE, W. E., ADAMS, J. A., \& KIESS, H. $O$. lorgetting and natural language mediation. Journal of Experimental Psychology, 1966, 72, 829-833.

OLTON, R. M. The effect of a mnemonic upon the retention of paired associate verbal material Unpublished doctoral dissertation, University of California, Berkeley, 1966.

ROCK, I. The role of repetition in associative lcarning. American Joumal of Psychology, 1957, 70, 186-193.

SMITH, K., \& NOBLE, C. E. Effects of a mnemonic technique applied to verbal learning and memory. Perceptual \& Motor Skills, 1965 , 21. 123-134.
IHURSTONE, L. L. The relation between learning time and length of task. Psychological Review, $1930,37,44-53$.

WNLLACLE, W. H., TURNIR. S. H., \& PERKINS,
C. C. Preliminary studies of human information storage. D.A. Project No. 3-99-12-023. S.C. Project No. 1320. University of Pennsylvania, 1957.

\section{Do children really prefer visual complexity?}

CORINNE HUTT and PENNY L. MCGREW,
Human Development Research Unit,
University of Oxford, Oxford, England

Children, 5, 8, and 11 years of age, were given the choice of exposing for themselves simple or complex pattems to view. In the case of random polygons, more simple than complex figures were exposed and contrariwise in the case of stimuli taken from Berlyne's previous studies; in both cases, the differences were insignificant. Viewing times generally decreased with age, 5-year-olds, however, viewed simple figures longer than complex ones, 11-year-olds vice versa, and 8-year-olds showed no difference. The Age by Complexity interaction was discussed in terms of the dimensions of "interestingness" and "pleasingness"

In many recent studies of visual attention and exploration, preference (for complexity) has been equated with amount of fixation. In other words, those stimuli viewed longer have been regarded as preferred stimuli (e.g., Smock \& Holt, 1962; Hershenson, Munsinger, \& Kessen, 1965; Thomas, 1966). A priori there seems little justification for such an assumption. The fact that complex stimuli (i.e., those with more detail) are viewed longer than simple ones may simply mean that an individual needs to fixate or scan these stimuli more in order to identify and categorize them (Berlyne, 1958). Furthermore, recent studies have demonstrated a disjunction between the dimensions of "interestingness" and "pleasingness" (Day, 1966, 1967; Berlyne, Ogilvie, \& Parham, 1968), symmetry being more closely associated with the latter and complexity with the former.

To be satisfactory, therefore, a preference measure should involve a choice on the part of the $\mathrm{S}$-either to view a particular stimulus again or to categorize preferentially. If there is a real preference for certain stimulus attributes, then these attributes should be capable of acting as reinforcers in an operant task where one of a pair of discriminanda controlled the appearance of these stimulus characteristics.

\section{METHOD}

To test this hypothesis, a modified teaching machine (Educational Systems,
Ltd., Model 1024) was used. Each of two buttons controlled exposures of alternate frames on a $35 \cdot \mathrm{mm}$ film strip. The stimulus material consisted of five series of pairs of figures, four of which were from Berlyne (1958), representing irregularity of arrangement, amount of material, heterogeneity of elements, and irregularity of shape. There were four pairs in each of these series, one member of each pair being relatively simpler (S) than the other $(\mathrm{C})$. The fifth series consisted of randomly generated figures (Attneave \& Amoult, 1956; Munsinger \& Kessen, 1964), the $C$ members consisting of four figures each of $5,10,15$, and 20 independent turns; the $S$ members of this consisted of symmetrical 5-, 10, 15 and 20-tum figures. The patterns were produced by fitting black paper cutouts to an outline on white card. These patterns were then photographed onto $35-\mathrm{mm}$ film, the 32 pattems from Berlyne's material on the first 32 frames in a randomized sequence, with the single constraint that the $S$ members occupied odd-numbered frames and the $C$ members occupied the even-numbered ones. These 32 patterns were photographed in reverse order on the next 32 frames. Similarly, Frames 65 to 96 contained the 32 random polygon patterns whose reverse order occupied Frames 97 to 128. If the two response buttons were pressed in strict alternation, simple and complex members of successive pairs were exposed alternately; if the same button was pressed repeatedly, the simple or complex members (as the case might be) of successive pairs were exposed.

\section{SUBJECTS}

The Ss were 24 5-, 8-, and 11-year-old children, eight in each age group, with equal numbers of boys and girls.

The Ss were told that they could play with a new machine and see different pictures by pressing the buttons; they could look at each picture for as long as they wished and had to press one of the buttons when they wanted to see a new one. Six trial frames at the beginning of the film helped the Ss to get used to operating the machine. PROCEDURE

The 64 pictures were presented in four runs: (1) on Run 1 only the first 32 were available, the left button (LB) controlling the $\mathrm{S}$ pictures and the $\mathrm{RB}$ controlling the $\mathrm{C}$ 